\title{
Editorial
}

Digestion

\section{Vancomycin in Very-Early Onset Inflammatory Bowel Disease-Dysbiosis: Fight Fire with Fire?}

\author{
Luc Biedermann \\ Division of Gastroenterology and Hepatology, University Hospital Zurich, Zurich, Switzerland
}

"Inflammatory bowel disease (IBD) refers to a group of chronic inflammatory disease of the gastrointestinal tract that is characterized by an inadequate immune response, modulated by genetics and environmental factors against the commensal intestinal microbiota." A sentence resembling the former represents a frequently used introduction for any scientific paper on IBD to outline the current concept of IBD. Indeed, the intestinal microbiota nowadays is among the most frequently mentioned pathophysiological hallmarks, whenever the current understanding of disease pathogenesis is discussed. In line with this, we are being confronted with an increasing number of original publications and review papers on microbial composition in IBD patients, focusing on alterations as compared to non-IBD subjects.

Yet, in striking contrast to the increasing amount of descriptive literature as well as a general rise in the general interest in this topic by both clinical and scientific gastroenterologists, there is a paucity of therapeutic approaches to target the well-established dysbiosis in IBD. The vast majority of current and evolving therapeutic targets address the inadequately stimulated immune response aiming at de-activating pro-inflammatory cascades. In relation to the plethora of already available and emerging substances, the amount of microbiota-manipulating treatment strategies under development and the financial backbone to support such developments are rather negligible. Among the few exceptions is fecal mi-

\section{KARGER}

๑) 2017 S. Karger AG, Basel

E-Mail karger@karger.com

www.karger.com/dig crobiota transplantation (FMT), which has revealed promising results in some $[1,2]$ but not all $[3]$ of the first high-quality trials in ulcerative colitis, whereas the currently available evidence in Crohn's disease [4] has to be considered insufficient. FMT represents a rather drastic microbiota-modulating-approach, where the jury - despite an increase in reassuring real-life data from several hundreds if not thousands on ultimate long-term safety still has to consider this approach. While first initiatives on shaping the future on "Next-Generation Microbiota Therapeutics" emerge [5], one might forget that we already have a large armamentarium of established, safe, effective, low-priced and generally available microbiotamodulating agents of variable specificity in our hands antibiotics.

The available evidence on the efficacy in IBD is considerably limited and the number of included patients in high-quality double-blind randomized trials is small [6]. This may be one reason why their general role in the treatment of both Crohn's disease (aside from penetrating complications or prophylaxis of postoperative recurrence) and ulcerative colitis (aside from pouchitis) is negligible, which is also reflected in established treatment guidelines [7, 8]. The paucity of available data is even more of an issue in very-early onset IBD. And this is the case, even if it appears plausible to assume that aside from genetic factors, the contribution of intestinal dysbiosis which may be a direct consequence of a distinct gene- 
mediated immune deficiency - fuels the inflammatory fire, it may be of utmost importance in the very early onset forms of the disease. One potential underlying reason for this restriction might be due to the concerns driven by a robust body of epidemiological evidence, suggesting an increased risk of developing IBD in the future, subsequent to infant exposure to antibiotics $[9,10]$, which might potentially even be correlated to the number of courses of antibiotics received [11]. Would antibiotics addressing intestinal dysbiosis in very early onset IBD thus represent a "fight-fire-with-fire" approach?

In this issue of the journal, Lev-Tzion et al. [12] report on their experience with vancomycin and gentamycin to treat severe and refractory early onset IBD. In a case series of 5 patients with a very early onset of their disease (mean age 1.6 years) with refractory disease, a considerable therapeutic success with an agent, dedicated in covering the gram-positive spectrum of the intestinal microbiota, could be achieved in 4 out of 5 patients. These results were obtained with an established antibiotic agent harboring and important advantage as opposed to numerous other candidate regimens. It has a nearly absent systemic absorption [13], aside from a limited systemic exposure in severely ill patients in the ICU or patients with advanced renal failure [14].

Although findings are derived from an uncontrolled series with only such a small sample size, they can never be considered a basis for advocating a wider clinical adaptation. The work by this Israelian group is important in that it may stimulate further research on this topic and potentially on other antibiotic agents or even combinations of these in the treatment of IBD, be it in the adult, children or even very early-onset population. These attempts might reveal to be even more promising if the pretreatment patient's microbiota composition could be taken into consideration, which might become a realistic option in view of the increasing dissemination of information and falling costs of high-throughput-sequencing approaches to characterize intestinal microbial composition.

\section{References}

1 Moayyedi P, Surette MG, Kim PT, et al: Fecal microbiota transplantation induces remission in patients with active ulcerative colitis in a randomized controlled trial. Gastroenterology 2015;149:102.

2 Paramsothy S, Kamm MA, Kaakoush NO, et al: Multidonor intensive faecal microbiota transplantation for active ulcerative colitis: a randomised placebo-controlled trial. Lancet 2017;389:1218-1228.

3 Rossen NG, Fuentes S, van der Spek MJ, et al: Findings from a randomized controlled trial of fecal transplantation for patients with ulcerative colitis. Gastroenterology 2015;149: 110-118.e4.

4 Vaughn BP, Vatanen T, Allegretti JR, et al: Increased intestinal microbial diversity following fecal microbiota transplant for active Crohn's disease. Inflamm Bowel Dis 2016;22: 2182-2190.
5 Petrof EO, Khoruts A: From stool transplants to next-generation microbiota therapeutics. Gastroenterology 146:1573-1582.

6 Khan KJ, Ullman TA, Ford AC, et al: Antibiotic therapy in inflammatory bowel disease: a systematic review and meta-analysis. Am J Gastroenterol 2011;106:661-673.

7 Dignass A, Lindsay JO, Sturm A, et al: Second European evidence-based consensus on the diagnosis and management of ulcerative colitis part 2: current management. J Crohns Colitis 2012;6:991-1030.

8 Gomollón F, Dignass A, Annese V, et al: 3rd European evidence-based consensus on the diagnosis and management of Crohn's disease 2016: part 1: diagnosis and medical management. J Crohn's Colitis 2017;113-25.

9 Shaw SY, Blanchard JF, Bernstein CN: Association between the use of antibiotics and new diagnoses of Crohn's disease and ulcerative colitis. Am J Gastroenterol 2011;106: 2133-2142.
10 Kronman MP, Zaoutis TE, Haynes $\mathrm{K}$, et al Antibiotic exposure and IBD development among children: a population-based cohort study. Pediatrics 2012;103:794-803.

11 Hviid A, Svanström H, Frisch M: Antibiotic use and inflammatory bowel diseases in childhood. Gut 2011;60:49-54.

12 Lev-Tzion R, Ledder O, Shteyer E, Tan MLN, Uhlig $\mathrm{HH}$, Turner D: Oral vancomycin and gentamicin for treatment of very early onset inflammatory bowel disease. Digestion 2017; 95:310-313.

13 Rao S, Kupfer Y, Pagala M, et al: Systemic absorption of oral vancomycin in patients with Clostridium difficile infection. Scand J Infect Dis 2011;43:386-388.

14 Pettit NN, DePestel DD, Fohl AL, et al: Risk factors for systemic vancomycin exposure following administration of oral vancomycin for the treatment of Clostridium difficile infection. Pharmacotherapy 2015;35:119-126. 